\title{
Significant Difference in the Serum Aspartate Aminotransferase Levels Across the Diagnostic Categories of Various Cardiac Diseases
}

\author{
Smita Deokar ${ }^{1}$, Sucheta Dandekar ${ }^{2}$
}

\author{
${ }^{1}$ Department of Biochemistry, HBT Medical College and Dr. R. N. Cooper hospital, Mumbai, Maharashtra, India, \\ ${ }^{2}$ Department of Biochemistry, Era University, Era's Lucknow Medical College and hospital, Lucknow, Uttar Pradesh,
}

India

\begin{abstract}
Background: Aspartate aminotransferase (AST), which generally reflects liver disease severity, has been associated with increased risk of cardiovascular disease (CVD). Aim: This study aimed to explore the relationship between risk factors such as serum AST and across the various diagnostic categories of cardiac diseases. Materials and Methods: We recruited 227 cases of CVDs (complete heart block [CHB]-16, heart failure [HF]-22, myocardial infarction-169, and rheumatic heart disease [RHD]-20) who were admitted in cardiology ward which were taken as cases and age- and gender-matched healthy persons were taken as controls $(n=228)$. Serum AST activity estimation was performed using the commercially available kit on fully automated chemistry analyzer. Data are presented as median and interquartile range. Statistical analysis was done on SPSS 16.0 and Microsoft Excel. Difference in the level of AST across the diagnostic categories was calculated by Kruskal-Wallis test. $P<0.05$ was taken as statistically significant. Results and Conclusion: Statistically significant difference in the AST levels was observed across the diagnostic categories. The post-hoc Dunn test showed a significant difference in the mean of AST levels between cases with CHB and HF $(P=0.0018)$ and between cases of RHD and $\mathrm{HF}(P=0.0004)$. In both cases, the median AST levels were higher in HF than in CHB and in RHD.
\end{abstract}

Key words: Aspartate transaminase, biomarker, cardiac risk factor, cardiovascular disease, heart failure, myocardial infarction

\section{INTRODUCTION}

Cardiovascular diseases (CVDs) are the leading cause of death all over the world. ${ }^{[1,2]}$ Over consumption of fats or genetic reason has increased the risk of CVDs day by day which is the leading cause of morbidity and mortality from infancy to old age. Even though traditional risk factors are available for diagnosis of CVD, still there is a lacuna of authentic and accurate biomarkers which had led to

\begin{tabular}{|c|c|}
\hline \multicolumn{2}{|c|}{ Access this article online } \\
\hline Publisher & \multirow[b]{2}{*}{$\begin{array}{l}\text { Website: } \\
\text { www.ijdms.in }\end{array}$} \\
\hline & \\
\hline & DOI: 10.30954/IJDMS.1.2020.5 \\
\hline
\end{tabular}

delayed diagnosis and treatment of patients. Hence, it is very important to identify the risk factors as early as possible and treatment should be started accordingly. ${ }^{[3]}$

Aspartate aminotransferase (AST) is the intracellular enzyme responsible for the catalysis of transamination between amino acids and ketonic acid. Increased AST usually indicates liver injury which is widely used in clinical practice to evaluate liver function. ${ }^{[4]}$ This liver function test enzyme is example of emerging biomarkers for CVD risk. ${ }^{[1]} \mathrm{CVD}$ is also the leading cause of death in non-alcoholic fatty liver disease, with higher rates coinciding with higher liver-related mortality over follow-up periods from 10 to 20 years. ${ }^{[5]}$ Monocytes of heart, liver, lung, bone, muscle, kidney and pancreas, and hepatocytes have the highest expression of AST, thus it is widely distributed in the heart. ${ }^{[4]}$ This aminotransferase enzyme is released into the blood in greater amounts when there is damage to the tissue cell membrane resulting in

\footnotetext{
Address for Correspondence:

Department of Biochemistry, HBT Medical College and Dr. R.N. Cooper Hospital, Mumbai - 400 056, Maharashtra, India
}

Submission: 02 July 2020; Revision: 16 July 2020; Acceptance: 20 July 2020 
increased permeability. ${ }^{[6]}$ There is increasing interest in using liver enzyme like AST as novel markers of cardiovascular risk. Even though now AST is not measured for CVD diagnosis, rising evidence showed that increased AST activity increases CVD risk and its mortality too. ${ }^{[7]}$

At present, there are very inadequate numbers of studies related to AST activity as a cardiac risk factor or cardiac marker in CVDs. Therefore, we have analyzed serum AST activity in various cardiac diseases and also observed the difference in level across various diagnostic categories, that is, myocardial infarction (MI), heart failure (HF), rheumatic heart disease (RHD), complete heart block (CHB), myocarditis, and coronary heart disease (CHD) patients. With our existing knowledge, this is the first study in Western Maharashtra population, particularly in Mumbai city.

\section{MATERIALS AND METHODS}

In this study, 227 subjects of CVDs (CHB-16, HF-22, MI169, and RHD-20) who were enrolled at cardiology and medicine department at a tertiary care hospital, Mumbai, India. Along with that, 228 age- and sex-matched healthy controls (controls without heart disease but with diabetes mellitus $[\mathrm{DM}]$ and hypertension $[\mathrm{HT}]=121$ and controls without heart disease, DM and HT $=107$ ) were included in a case-control study. The study was approved by the Institutional Ethics Committee. All the cases were diagnosed by cardiologists and final patient selection was done. Diagnostic test done was two-dimensional echo along with electrocardiogram and final selection of the patients was done with questionnaire to patient. Mean age for cases was 54.49 years and for controls 54.03 years. Patients with chronic illnesses such as liver diseases, bone diseases, pregnant woman, malignancies, infections, and rheumatic arthritis were excluded where the level of AST is presumed to be raised. Serum AST activity estimation was done by modified International Federation of Clinical Chemistry method (kits by Pathozyme Diagnostics) using the commercially available kit on fully automated chemistry analyzer. Statistical analysis was carried out using SPSS version 16.0 and Microsoft Excel.

\section{RESULTS}

Table 1: Age description of cases and controls

\begin{tabular}{llcccc}
\hline $\begin{array}{l}\text { Subject } \\
\text { group }\end{array}$ & Mean & $\begin{array}{l}\text { Standard } \\
\text { deviation }\end{array}$ & Minimum & Maximum & $\begin{array}{c}\text { P value (Mann- } \\
\text { Whitney } \\
\text { U-test) }\end{array}$ \\
\hline Cases & 54.49 & 11.71 & 24 & 83 & 0.636 \\
Controls & 54.03 & 12.35 & 23 & 80 & \\
\hline
\end{tabular}

\section{DISCUSSION}

The study included 227 cases and 228 age- and sex-matched controls.
The age distribution of the cases is shown in Table 1. The cases and controls were age matched $(P=0.636)$. The mean (standard deviation) age of cases was 54.49 years and that of controls was 54.03 years. Cases were in the range of 24-83 years and controls between 23 and 80 years.

The median (interquartile range) level of serum AST as a cardiac risk factor was significantly raised in cases $(P<0.0005) 46$ (29.75) IU/L than the controls (HT and DM) 23 (8) IU/L and in controls (without HT and DM) 23 (6) IU/L [Table 2]

It is observed from the above table that there is a statistically significant difference in the AST levels across the diagnostic categories. Therefore, the post hoc test, namely, the Dunn test was run for this variable [Table 3].

The post hoc test shows a significant difference in the mean of AST levels between cases with CHB and HF $(P=0.0018)$ and between cases of RHD and HF $(P=0.0004)$. The median AST levels were higher in HF than in CHB and in RHD [Tables 4 and 5].

Our study supports and expands previous studies that reported an association between serum AST activities with the presence and severity of angiographically documented premature CAD. ${ }^{[7]}$ Shen et al. in 2015 found out that the serum levels of AST in CHD patients are higher than those in controls. Thus, the high serum AST (a biochemical marker) can be used to predict the severity of CHD and is also proved as an independent risk factors of CHD. ${ }^{[4]}$ Weng et al. in 2015 found out an elevated AST/ALT ratio which is significantly associated with increased risk of developing CVD in men but not women. The AST/ALT ratio being associated with CVD in men may be related to higher prevalence of liver disease in men..$^{[1]}$

The association of a raised AST with CVD may have several explanations:

1. As there is an increasing body of evidence in support of the relationship between elevated liver transaminases with BP, dyslipidemia, and blood glucose as the part of the metabolic syndrome that could be a major risk factor for CAD. ${ }^{[8]}$

2. As, it is usually known that inflammation and oxidative stress are key components of atherosclerosis. ${ }^{[7]}$

3. AST is widely distributed in the heart, liver, lung, bone, muscle, kidney, and pancreas, of which myocytes have the highest expression of AST, followed by hepatocytes. Exists in two isoforms in myocytes: Cytoplasmic AST and mitochondrial, that is, m-AST. In mitochondria, about $80 \%$ of AST exists, and so, it is a non-specific intracellular functional enzyme. Usually, the serum AST is at a low. Whenever there is myocardial injury, mitochondria are damaged, and serum m-AST increases significantly 
Table 2: Serum AST among cases and controls

\begin{tabular}{|c|c|c|c|c|}
\hline Parameter (median [IQR]) & Cases (mg/dL) & $\begin{array}{c}\text { Controls without heart disease } \\
\text { but with } \mathrm{DM} \text { and } \mathrm{HT}=121\end{array}$ & $\begin{array}{c}\text { Controls without heart } \\
\text { disease, DM and } \mathrm{HT}=107\end{array}$ & $P$ value \\
\hline Serum AST IU/L $(n=448$, Cases=220) & $46(29.75)$ & $23(8)$ & $23(6)$ & $<0.0005^{\star}$ \\
\hline
\end{tabular}

AST: Aspartate aminotransferase, IQR: Interquartile range, DM: Diabetes mellitus, HT: Hypertension

Table 3: Difference in the level of serum AST across the diagnostic categories (MI, CHB, HF, and RHD). (Kruskal-Wallis test)

\begin{tabular}{lccc}
\hline Marker & Chi-square & df & Asymp. sig. \\
\hline AST & 20.1 & 3 & $<0.0005^{*}$ \\
\hline
\end{tabular}

* Significant at 0.05 level. AST: Aspartate aminotransferase, CHB: Complete heart block, HF: Heart failure, MI: Myocardial infarction, RHD: Rheumatic heart disease

Table 4: Post hoc Dunn test for AST

\begin{tabular}{lllcc}
\hline $\begin{array}{l}\text { Dependent } \\
\text { variable }\end{array}$ & $\begin{array}{l}\text { (I) } \\
\text { Diagnosis }\end{array}$ & $\begin{array}{l}\text { (J) } \\
\text { Diagnosis }\end{array}$ & $\begin{array}{c}\text { Z versus } \\
\text { critical value }\end{array}$ & Sig. \\
\hline AST & MI & CHB & $2.63630 \geq 2.128$ & 0.0084 \\
& & HF & $1.48326<2.128$ & 0.13801 \\
& & RHD & $3.22113 \geq 2.128$ & 0.0013 \\
& CHB & MI & $2.63630 \geq 2.128$ & 0.0084 \\
& & HF & $3.12136 \geq 2.128$ & $0.0018^{*}$ \\
& HF & RHD & $0.20134<2.128$ & 0.84043 \\
& & CHB & $1.48326<2.128$ & 0.13801 \\
& & RHD & $3.12136 \geq 2.128$ & $0.0018^{*}$ \\
& RHD & MI & $3.22113 \geq 2.128$ & 0.0013 \\
& & CHB & $0.20134<2.128$ & 0.84043 \\
& & HF & $3.55234 \geq 2.128$ & $0.0004^{*}$ \\
\hline
\end{tabular}

${ }^{*}$ Comparisons: 6 , Ties: 75 , adjusted for ties. Family alpha: 0.2,

Bonferroni individual alpha: 0.033, Bonferroni Z-value (two sided): 2.128. AST: Aspartate aminotransferase, CHB: Complete heart block, HF: Heart failure, MI: Myocardial infarction, RHD: Rheumatic heart disease

Table 5: Median levels of AST for each class

\begin{tabular}{lccc}
\hline Marker & Diagnostic class & Median & Interquartile range \\
\hline AST & MI & 48 & 30 \\
& $\mathrm{CHB}$ & 30 & 26.5 \\
& $\mathrm{HF}$ & 51 & 58.5 \\
& $\mathrm{RHD}$ & 33 & 18 \\
\hline
\end{tabular}

AST: Aspartate aminotransferase, CHB: Complete heart block, HF: Heart failure, Ml: Myocardial infarction, RHD: Rheumatic heart disease

where further serum m-AST may reflect the severity of myocyte injury. ${ }^{[4]}$

4. Advanced liver dysfunction may have a negative impact on renal function by way of splanchnic vasodilatation resulting in arterial underfilling and renal vasoconstriction. Hence, in chronic HF patients, liver congestion may directly contribute to diminished natriuresis. ${ }^{[9]}$

\section{CONCLUSION}

Regardless of the underlying mechanisms, our results suggest that AST would be useful biomarkers for cardiovascular risk evaluation which can be an important biomarker and indicator of future HF. Regarding the availability and simplicity of AST in routine clinical practice and their universal standardization, these tests have possibility to be considered as an additional marker for CVD outcomes.

\section{REFERENCES}

1. Weng SF, Kai J, Guha IN, Qureshi N. The value of aspartate aminotransferase and alanine aminotransferase in cardiovascular disease risk assessment. Open Heart 2015;2:e000272.

2. Smita D, Dandekar S, Chaturvedi S. Increased serum alkaline phosphatase activity in different cardiac diseases. Int J Recent Sci Res 2018;9:24262-4.

3. Upadhyay RK. Emerging risk biomarkers in cardiovascular diseases and disorders. J Lipids 2015;2015:971453.

4. Shen J, Zhang J, Wen J, Ming Q, Zhang J, Xu Y. Correlation of serum alanine aminotransferase and aspartate aminotransferase with coronary heart disease. Int J Clin Exp Med 2015;8:4399404.

5. Cecilia S, Hultcrantz R. Decreased survival of subjects with elevated liver function tests during a 28-year follow-up 1. Hepatology 2010;51:595-602.

6. Kasper DL, Braunwald E, Fauci H. Harrison's Principles of Internal Medicine. $16^{\text {th }}$ ed. New York: McGraw-Hill; 2004. p. 1301-486.

7. Masoudkabir F, Karbalai S, Vasheghani-Farahani A, Aliabadi LL, Boroumand MA, Aiatollahzade-Esfahani F, et al. The association of liver transaminase activity with presence and severity of premature coronary artery disease. Angiology 2011;62:614-9.

8. Han X, Boisvert WA. Interleukin-10 protects against atherosclerosis by modulating multiple atherogenic macrophage function. Thromb Haemost 2015;113:505-12.

9. Cagli K, Basar FN, Tok D, Turak O, Basar O. How to interpret liver function tests in heart failure patients? Turk J Gastroenterol 2015;26:197-203.

How to cite this article: Deokar S, Dandekar S. Significant Difference in the Serum Aspartate Aminotransferase Levels Across the Diagnostic Categories of Various Cardiac Diseases. Int J Dent Med Spec 2020;7(1):26-28.

Source of Support: None; Conflicts of Interest: None 